\title{
DETERMINACIÓN DE Echinococcus granulosus EN PERROS DEL CONO NORTE DE LIMA
}

\author{
Julio Chuquisana A. ${ }^{1}$, Amanda Chávez V. ${ }^{2}$ y Eva Casas A. ${ }^{2}$
}

\section{Abintet}

The objective of this study was to determine the presence of Echinococcus granulosus in the dog population of northern Lima. A total of 600 dogs from the districts of Rímac, San Martin de Porres, Independencia, Los Olivos, Carabayllo, Comas, San Juan de Lurigancho and Puente Piedra were tested. Half the dogs $(n=300)$ were purged with arecolina bromide and half submitted to macroscopic evaluation of the thin intestine. Only one dog out of the 600 was found to be positive $(0.3 \pm 0.06 \%)$, however given the poor sanitation and hygiene conditions of the San Juan de Lurigancho district where it was found, its presence represents a potential health risk for the human population. Periodic testing to determine the presence of $E$. granulosus in this district, and other marginal regions of greater Lima, should be carried out. The incidence of infection, however, has remained essentially unchanged since the last tests were performed in Lima 27 years ago.

Key words: Dogs, Echinococcus, equinococosis, zoonosis.

\section{Resumen}

El objetivo de este estudio fue determinar la presencia de Echinococcus granulosus en perros $(n=600)$ del cono norte de Lima mediante la utilización de dos técnicas de diagnóstico la purga con bromahidrato de arecolina $(n=300)$ y la evaluación macroscópica del intestino delgado $(n=300)$ para identificar al parásito de perros provenientes de los distritos del cono norte (Rímac, San Martín de Porres, Independencia, Los Olivos, Carabayllo. Comas, San Juan de Lurigancho, Puente Piedra). Mediante la técnica de purga con bromhidrato de arecolina se halló un perro positivo a Echinococcus granulosus $(0.3 \pm 0.06 \%)$ perteneciente al distrito de San Juan de Lurigancho. Ningún perro evaluado mediante la técnica macroscópica del intestino delgado presentó Echinococcus granulosus. Este resultado, sumado a las deficientes condiciones higiénico-sanitarias, bajos niveles socioeconómicos y culturales de la población que intervienen como factores importantes en la cadena de transmisión y persistencia de la infección, constituyen un foco infeccioso de riesgo potencial para la salud de la población. Por este motivo se debería realizarse monitoreos periódicos en caninos a nivel de éste distrito, como en otros urbano marginales. Por otro lado la prevalencia obtenida de $0.3 \pm 0.06 \%$ indicaría que la enfermedad se mantiene en el cono norte de Lima, igual a los niveles hallados hace 27 años en Lima Metropolitana.

Palabras clave: Perros, Echinococcus, equinococosis, zoonosis.

\footnotetext{
' Práctica privada

${ }^{2}$ Laboratorio de Parasitologia - FMV - UNMSM. E-mail: d170010@unmsm.edu.pe
} 


\section{Introduccion}

La equinococosis, es una zoonosis parasitaria causada por el metacéstodo del Echinococcus granulosus, cuyo hospedero definitivo y principal diseminador, es el perro. La convivencia de este cánido con el ser humano, las deficientes condiciones higiénico-sanitarias, bajos niveles socioeconómicos y culturales de la población, intervienen como factores importantes en la cadena de transmisión y persistencia de la infección.

La equinococosis ocasiona grandes pérdidas económicas en la ganadería por el decomiso de órganos afectados y la baja productividad de los animales, sobre todo en la crianza extensiva del ganado ovino. Las zonas de la sierra central y sur del Perú, reúnen las características ecológicas, culturales, económicas y sociales que permiten el mantenimiento del ciclo de vida del metacéstodo (quiste hidatídico), siendo estas áreas reportadas como endémicas no sólo de hidatidosis ovina sino también de hidatidosis humana.

En el mundo, América Latina es considerada como una de las áreas de mayor prevalencia de equinococosis (Schantz, 1972), siendo Argentina, Brasil, Chile, Uruguay y Perú los que registran tasas de infección más elevadas (Neghme, 1985). En el Perú se han determinado los focos de hidatidosis mediante el diagnóstico de la infección a la inspección post mortem en camales, y en los perros mediante campañas de eliminación. El uso del bromohidrato de arecolina como tenífugo; surgió como una alternativa de diagnóstico en la década del 70 . Basándose en estas técnicas se han obtenido los datos de prevalencia en nuestro país, no obstante las dificultades y riesgos que conlleva su empleo por la manipulación de los excrementos contaminados. Sin embargo, su utilidad se considera importante en la evaluación de los programas de control de la enfermedad.
En el Perú, en ciudades de la costa las prevalencias reportadas para la infección con E. granulosus es baja como lo demuestran los estudios de Acha (1952) Inope y Torres (1972), Cosios (1972), Medina (1972), quienes reportaron una prevalencia no mayor al $0.94 \%$. Luego, Mori (1974) encontró en el distrito de Lurigancho (Chosica) una prevalencia del $2.6 \%$ de un total de 112 animales. En otro estudio realizado en los alrededores del camal de Chincha, Cabrera (1998) encontró una prevalencia de $6.25 \%$ de un total de 48 animales.

Según datos emitidos por el Ministerio de Salud, en la ciudad de Lima los casos de hidatidosis humana se han incrementado durante el período 1993-98. Esto, podría deberse a las mejoras en las técnicas de diagnóstico. Sin embargo, los casos autóctonos encontrados se podría explicar por la migración hacia la capital de pobladores de áreas rurales que traen consigo algunos animales como ovinos y perros generalmente infectados con quistes hidatídicos y Echinococcus. Por lo que el objetivo de este estudio fue determinar la presencia de E. granulosus en perros del cono norte de Lima, mediante dos técnicas de diagnóstico para identificar al parásito de perros provenientes de los distritos del cono norte (Rímac, San Martín de Porres, Independencia, Los Olivos, Carabayllo, Comas, San Juan de Lurigancho y Puente Piedra).

\section{Materiales y Metodos}

El trabajo se realizó con la colaboración del Centro Antirrábico de Lima y el Centro de Salud Ambiental DISUR III - Lima Norte, instituciones que realizan el diagnóstico de rabia y monitoreos contínuos de rabia mediante atrape y sacrificio de perros vagos. El mayor porcentaje de estos animales provienen del Cercado de Lima, Callao y del Cono Norte (Rímac, San Martín de Porres, 
Independencia, Los Olivos, Carabayllo, Comas, San Juan de Lurigancho, Puente Piedra).

El tamaño muestreal fue obtenido mediante el método de prevalencia límite del 1\% y un nivel de confianza del $95 \%$, habiéndose obtenido un total de 600 muestras y cuya evaluación se realizó en el laboratorio de Parasitología de la Facultad de Medicina Veterinaria de la UNMSM.

\section{Métodos de Diagnóstico}

\section{- Evaluación macroscópica del intes- tino delgado.}

Luego del sacrificio de los perros, se aisló el intestino delgado que fue ligado por ambos extremos, inyectándose una solución tibia de bicarbonato de sodio al $20 \%$ con el fin de facilitar el desprendimiento de los parásitos de la mucosa intestinal. Una hora después se inyectó una solución de formol al $10 \%$ y fue hervido por 10 minutos guardado en refrigeración hasta el momento de ser trabajado. La técnica a seguir para la identificación del parásito es la descrita por Cosios (1972).

\section{- Purga con bromohidrato de arecolina}

Los perros en cuarentena del Centro de Salud Ambiental DISUR III Lima - Norte, fueron tratados con bromohidrato de arecolina (tenífugo) en dosis de $3.5 \mathrm{mg} /$ $\mathrm{kg}$ de peso vivo para provocar el desprendimiento de las tenias de las paredes del intestino junto con una evacuación semi-diarreica. Los perros fueron colocados en caniles de fácil limpieza donde se colectaban las heces y los parásitos. La porción mucosa de las heces evacuadas se pasaban por tamices para realizar la búsqueda e identificación de los parásitos en forma visual y en base a sus características morfológicas.

\section{Revilntos}

De las 300 muestras analizadas por purga con bromohidrato de arecolina se encontró un animal positivo a Echinococcus granulosus representando $0.3 \pm 0.06 \%$ (1/ 300). En tanto, que las otras 300 muestras evaluadas por sacrificio dieron resultados negativos a Echinococcus granulosus. (Cuadro 1).

Cuadro 1. Evaluación de Echinococcus granulosus en perros procedentes del cono norte de Lima Metropolitana, mediante dos técnicas diagnósticas, 1998.

\begin{tabular}{lccc}
\hline Técnicas diagnósticas & $\mathrm{N}^{\circ}$ de perros & $\begin{array}{c}\mathrm{N}^{\circ} \text { de perros } \\
\text { positivos }\end{array}$ & Porcentaje \pm I.C. \\
\hline $\begin{array}{l}\text { Purga con bromhidrato de } \\
\text { arecolina }\end{array}$ & 300 & 1 & $0,3 \pm 0,06$ \\
$\begin{array}{l}\text { Evaluación macroscópica } \\
\text { del intestino delgado }\end{array}$ & 300 & 0 & 0 \\
\hline Total & 600 & 1 & $0.16 \pm 0.06$ \\
\hline
\end{tabular}

De todos los distritos muestreados del cono norte de Lima Metropolitana (Rímac, San Martín de Porres, Independencia, Los Olivos, Carabayllo, Comas, San Juan de Lurigancho, Puente Piedra), sólo en el distrito de San Juan de
Lurigancho se halló un caso positivo a equinococosis canina, encontrándose un porcentaje de $2.63 \pm 1.6 \%(1 / 38)$ para este distrito. El animal positivo provenía del A.A.H.H. Cruz de Motupe, distrito de San Juan de Lurigancho. 


\section{Discusión}

La evaluación macroscópica del intestino delgado es considerado el método ideal y más específico para la determinación del $E$. granulosus en el perro, teniendo como limitante fundamental el elevado número de perros a sacrificar, debido al factor afectivo que se da entre el dueño y su mascota en la ciudad, dificultando la toma de muestras adecuadas y por ende una real evaluación de ésta infección parasitaria, además el procesamiento de las muestras conlleva a un gran riesgo zoonótico, tomando este tipo de evaluación un mayor tiempo por los cuidados sanitarios que se deben considerar.

En nuestro estudio no se logró encontrar mediante la evaluación macroscópica del intestino delgado evidencia clínica de esta parasitosis en los perros sacrificados. Sin embargo, dicho resultado negativo no es concluyente ya que otros estudios indican que perros infectados con tenias inmaduras, así como con cargas parasitarias menores a 50 tenias son difícilmente detectables aún en animales procedentes de zonas endémicas (Craig et al., 1997). Tal es el caso reportado por Allan et al. (1992), quien evaluó necropsias de perros en campo, procedentes de una zona endémica, hallando un animal libre de la infección. Estos estudios explicarían el resultado hallado a la necropsia aparentemente negativo y a la vez contradictorio del obtenido por Acha (1952), quien al realizar 100 necropsias en perros procedentes del centro antirrábico de Lima reportó un animal infectado con 100 tenias del E. granulosus.

Desde el año 1972, los estudios de equinococosis canina se basaron en hallazgos de la evaluación macroscópica del intestino delgado. Cosios (1972) reportó una prevalencia del $0.94 \%$ de 318 perros muestreados en los distritos de la margen derecha del río Rímac de la ciudad de Lima (San Martín de Porres, Independencia, Carabayllo, Rímac y Comas). Encontrándose 3 casos clínicos positivos a $E$. granulosus (dos perros de Comas y uno del Rímac). En contraste, en el presente trabajo empleando igual técnica de diagnóstico (evaluación macroscópica del intestino delgado) en estos distritos y adicionalmente de otros distritos del cono norte de Lima no se encontró ningún perro parasitado con $E$. granulosus. Cabe señalar que si bien la técnica de evaluación macroscópica del intestino delgado fue empleado con fines diagnósticos en estos estudios, la población a muestrear fueron obtenidas de perros con antecedentes de mordedores y llevados por sus dueños al Centro Antirrábico para su control y en algunos casos para su sacrificio sugiriendo que fueron mascotas criadas con ciertas condiciones sanitarias. En nuestro país, Cabrera (1998) reportó $2.08 \%$ de equinococosis canina en los alrededores del camal municipal de Chincha mediante la técnica de purga, observando que el factor social fundamental para la persistencia del $E$. granulosus es la alimentación de perros con vísceras infectadas con quistes hidatídicos por parte de los trabajadores del camal.

En relación con la técnica diagnóstica de la purga con bromohidrato de arecolina, aprobada en la década del 70 por la OMS, surgió como una alternativa que permite elegir la procedencia de los perros a ser evaluados, así como poder dosificar un mayor número de perros, a diferencia de la técnica de evaluación macroscópica del intestino delgado en que el número de perros a evaluar es el factor limitante. Siendo la desventaja principal de esta técnica el riesgo biológico que enfrenta el personal desde la obtención de las muestras hasta el procesamiento de las mismas.

El caso positivo a E. granulosus, encontrado mediante la técnica de la purga con arecolina corresponde a un perro cruzado, sexo hembra, mayor de 6 años y con una carga parasitaria de 6 tenias adultas. Por lo tanto, el hallazgo de un perro positivo en el presente trabajo, es de gran importancia porque representa que la prevalencia de equinococosis canina sea al menos del $1 \%$ en los diferentes distritos del cono norte de Lima. 
Finalmente, los resultados obtenidos en este trabajo exhiben la potencialidad de la infección hidatídica y equinococosis urbana, considerado desde ya un problema de gran importancia zoonótica que debe ser tomado en cuenta por las autoridades sanitarias toda vez que un sólo proglótido que se elimina por las heces contiene de 200 a 600 huevos capaces de infectar a cualquier persona que se encuentre en el entorno del ambiente donde se ubica el perro parasitado (Craig et al., 1996), pudiendo incluso abarcar mayores áreas o ciudades, debido a la carencia de un programa serio de control periódico de esta parasitosis en países como el nuestro considerados hiperendémicos (Thakur, 1999). Además los huevos pueden diseminarse en todas las direcciones hasta 80$170 \mathrm{~m}$ del lugar en que fueron depositados en un plazo de 10 días (Cordero et al., 1999).

\section{Recomendactones}

El Ministerio de Salud y/o las Municipalidades deben realizar anualmente un monitoreo sanitario en perros para la evaluación de la equinococosis en zonas urbano marginales; realizar campañas quimioprofi- lácticas en estas zonas en riesgo $y$; hacer efectivo un programa de educación sanitaria sobre esta zoonosis (desde colegios), para crear conciencia de su peligro para la salud pública.

\section{Literatura Citada}

1. Acha, P. 1952. Porcentaje de parásitos del Canis familiaris en la ciudad de Lima. Tesis bachiller Fac. Med. Vet. Univ. Nac. Mayor San Marcos. 35 p.

2. Allan, J.C.; P.S. Craig; J. García Noval; F. Mencos; Y. D. Wang; $H$. Wen; R. Znottringer; M. Rogan y Zeymle. 1992. Coproantigen detection for inmunodiagnosis echinococosis and teniasis in dogs and humans. Parasitology, 104:347-355.

3. Cabrera, M. 1998. Determinación de la prevalencia de echinococosis canina en la zona perimétrica del camal municipal de Chincha. Tesis bachiller Facultad de Medicina Veterinaria. Universidad Nacional San Luis Gonzaga de Ica. 39 p.

4. Cordero, M.; F. Rojo; A. Martínez; M. Sánchez; S. Hernández; I. Navarrete; P. Diez; H. Quiroz y M. Carvalho. 1999. Hidatidosis. Parasitosis Sistémicas. En: Parasitología Veterinaria. Ed. McGrawHill-Interamericana de España, S.A.U. p: 341-349.

5. Cosios, C. 1972. Prevalencia de equinococosis canina en los distritos de la margen derecha del río Rímac de la ciudad de Lima. Tesis bachiller Fac. Med. Vet. Univ. Nac. Mayor San Marcos. 49p.

6. Craig, P.S.; M.T. Rogan y J.C. Allan. 1997. Inmunodiagnosis of Echinococcus granulosus and a comparison of techniques for diagnosis of canine echinococosis. En: Compendium of cystic echinococcosis in Africa and middle eastern with special reference of Morocco. Eds F.L. Andersen, H. Ouhelli, M. Kachani. Provo: Brigham Young University. p. 85-118.

7. Craig, P.S.; M.T. Rogan y J.C. Allan. 1996. Deteccion, screening and community epidemiology of taeniid cestode zoonoses: cystic echinococosis, alveolar echinococosis and neurocysticercosis. Advances in parasitology 38: $170-215$.

8. Inope, L. y J. Torres. 1972. Estudio de la hidatidosis como problema de salud pública en Lima Metropolitana. En: Sociedad Peruana de Parasitología. X Congreso Internacional de Hidatidosis, 22-27 Octubre. Arequipa-Perú.

9. Medina, M. 1972. equinococosis canina en los distritos de Lima Oeste. Tesis bachiller Fac. Med. Vet. Univ. Nac, Mayor San Marcos. $44 \mathrm{p}$.

10.Mori, F. 1974. Prevalencia de equinococosis en el distrito de Lurigancho (Chosica). Tesis Bach. Fac. Med. Vet. UNMSM. 25 p.

11. Neghme, A. 1985. Enfoque epidemiológico de la hidatidosis. Bol. Oficina Sanitaria Panamericana; 102 (2): 175-80. 
12. Schantz, P. 1972. Guía para el empleo del bromhidrato de Arecolina en el diagnóstico de la Infección por Echinococcus granulosus en el perro. Boletín Chileno de Parasitología. 28: 81 - 90.

13. Thakur, A. 1999. Epidemiology of hydatid disease in South America. En:
Archivos Internacionales de la Hidatidosis. XIX International Congress of Hydatidology San Carlos de Bariloche, Rio Negro, Argentina. Internacional Eds. Larrieu E., Romeo S., Mercapide C. Vol. XXXIII p: 55-61. 